\title{
A very extended molecular web around NGC 1275
}

\author{
P. Salomé ${ }^{1}$, F. Combes ${ }^{1}$, Y. Revaz ${ }^{5}$, D. Downes ${ }^{2}$, A. C. Edge ${ }^{3}$, and A. C. Fabian ${ }^{4}$ \\ 1 LERMA, Observatoire de Paris, UMR 8112 du CNRS, 75014 Paris, France \\ e-mail: philippe.salome@obspm.fr \\ 2 Institut de Radio Astronomie Millimétrique, Domaine Universitaire, 38406 St. Martin d'Hères, France \\ 3 Institute of Computational Cosmology, Durham University, South Road, Durham, DH1 3LE, UK \\ ${ }^{4}$ IoA, Madingley Road, Cambridge, CB3 OHA, UK \\ ${ }^{5}$ Laboratoire d'Astrophysique, École Polytechnique Fédérale de Lausanne (EPFL), Observatoire, 1290 Sauverny, Switzerland
}

Received 12 November 2008 / Accepted 16 May 2011

\begin{abstract}
We present the first detection of $\mathrm{CO}$ emission lines in the $\mathrm{H} \alpha$ filaments at distances as far as $50 \mathrm{kpc}$ from the centre of the galaxy NGC 1275. This gas is probably dense $\left(\geq 10^{3} \mathrm{~cm}^{-3}\right)$. However, it is not possible to accurately determine the density and the kinetic temperature of this relatively warm gas $\left(T_{\text {kin }} \sim 20-500 \mathrm{~K}\right)$ with the current data alone. The amount of molecular gas in the filaments is large $-10^{9} M_{\odot}$ (assuming a Galactic $N\left(\mathrm{H}_{2}\right) / I_{\mathrm{CO}}$ ratio). This is $10 \%$ of the total mass of molecular gas detected in this cD galaxy. This gas has large-scale velocities comparable to those seen in $\mathrm{H} \alpha$. The origin of the filaments is still unclear, but their formation is very likely linked to the AGN positive feedback that regulates the cooling of the surrounding X-ray-emitting gas as suggested by numerical simulations. We also present high-resolution spectra of the galaxy core. The spatial characteristics of the double-peaked profile suggest that the molecular web of filaments and streamers penetrates down to radii of less than $2 \mathrm{kpc}$ from the central AGN and eventually feeds the galaxy nucleus. The mass of gas inside the very central region is $\sim 10^{9} M_{\odot}$, and is similar to the mass of molecular gas found in the filaments.
\end{abstract}

Key words. cooling flows - galaxies: individual: NGC 1275 - galaxies: ISM - galaxies: kinematics and dynamics

\section{Introduction}

The cD galaxy NGC 1275 lies at the centre of the Perseus cluster, the brightest galaxy cluster in the sky in X-rays. The galaxy is at a redshift of $0.01756\left(D_{\mathrm{A}}=72.6 \mathrm{Mpc} ; 1^{\prime \prime}\right.$ is $\left.350 \mathrm{pc}\right)$. Past detections of CO in NGC 1275 were mainly toward the centre of the galaxy (Lazareff et al. 1989; Mirabel et al. 1989; Reuter et al. 1993; Braine et al. 1995; Inoue et al. 1996; Bridges \& Irwin 1998; Lim et al. 2008). We also studied CO in filamentary structures all around the galaxy (inside $r \leq 10 \mathrm{kpc}$ ) and in three pointings at galactocentric distances as large as $r \sim 25 \mathrm{kpc}$ (Salomé et al. 2006, 2008a,b). The molecular gas coincides with the $\mathrm{H} \alpha$ filaments (e.g., Cowie et al. 1983; Conselice et al. 2001), within the hot gas seen in soft X-rays at $0.5 \mathrm{keV}$ (Fabian et al. 2006). These results were in good agreement with the $\mathrm{CO}$ observations of the central $15 \mathrm{kpc}$ made by Reuter et al. (1993) although the regions observed were not exactly the same. The central region was also observed with the VLA by Jaffe (1990) who detected extended $21 \mathrm{~cm}$ absorption in front of the radio source $3 \mathrm{C} 84$. The $\mathrm{HI}$ is elongated in a east-west direction like the $\mathrm{CO}$ and the inferred neutral hydrogen mass found is $\sim 5 \times 10^{9} \mathrm{M}_{\odot}$, that is, of the same order as the molecular gas mass $\left(\sim 10^{10} M_{\odot}\right)$ found in the same region from CO observations (Salomé et al. 2006). Irwin \& Bridges (2001) found a north-west/south-east extended continuum emission at $450 \mu \mathrm{m}$ and $850 \mu \mathrm{m}$ with SCUBA. This core-subtracted emission excess reveals a large amount of cold dust inside the central $\sim 20 \mathrm{kpc}\left(6 \times 10^{7} M_{\odot}\right.$ with $T_{\text {dust }} \sim 20 \mathrm{~K}$ and $\beta=1.3$ ).

In this paper, we describe a new search with the IRAM $30 \mathrm{~m}$ telescope for molecular gas in the $\mathrm{H} \alpha$ filaments at the largest radii from the centre of NGC 1275 (outside the already observed central region and as far as $50 \mathrm{kpc}$ ). We clearly identify molecular filaments in five regions (see Fig. 1):

(1) a $30 \mathrm{kpc}-$ long filament, running north-south, not previously observed in $\mathrm{CO}$ (regions $\mathrm{r} 1$ through $\mathrm{r} 4$ ). The largest radius at which we detect $\mathrm{CO}$ (region $\mathrm{r} 1$ ) is $50 \mathrm{kpc}$ (in projection) north of the centre of the galaxy;

(2) the southeast $\mathrm{H} \alpha$ filaments ( $\mathrm{r} 10, \mathrm{r} 11, \mathrm{r} 15)$. The distance between the end of this filamentary system (region r11) and the end of the north filament (r1) is $200^{\prime \prime}(70 \mathrm{kpc})$;

(3) a tangential filament to the north-east (r19, r20), where we have extended our previous $\mathrm{CO}$ observations;

(4) the northwest area, including the well-known horseshoe filament, was observed in regions $\mathrm{r} 5, \mathrm{r} 6, \mathrm{r} 8, \mathrm{r} 9$, and $\mathrm{r} 16$;

(5) the central region around 3C 84 was re-observed. Here, we made a high-sensitivity spectrum with high spectral resolution.

Section 2 presents the observations. Section 3 describes the new $\mathrm{CO}$ detections. Section 4 discusses the excitation conditions of the molecular gas at large radii around NGC 1275 and the possible origin of the very distant molecular filaments.

\section{Observations}

We used the IRAM $30 \mathrm{~m}$ telescope on Pico Veleta, near Granada, Spain. Observations were made in December 2007. Receivers were tuned to the $\mathrm{CO}(1-0)$ and $\mathrm{CO}(2-1)$ lines, redshifted to the adopted systemic velocity of NGC 1275 (113.280 and $226.559 \mathrm{GHz}$ ). We used two $512 \times 1 \mathrm{MHz}$ filters at $3 \mathrm{~mm}$ and two 


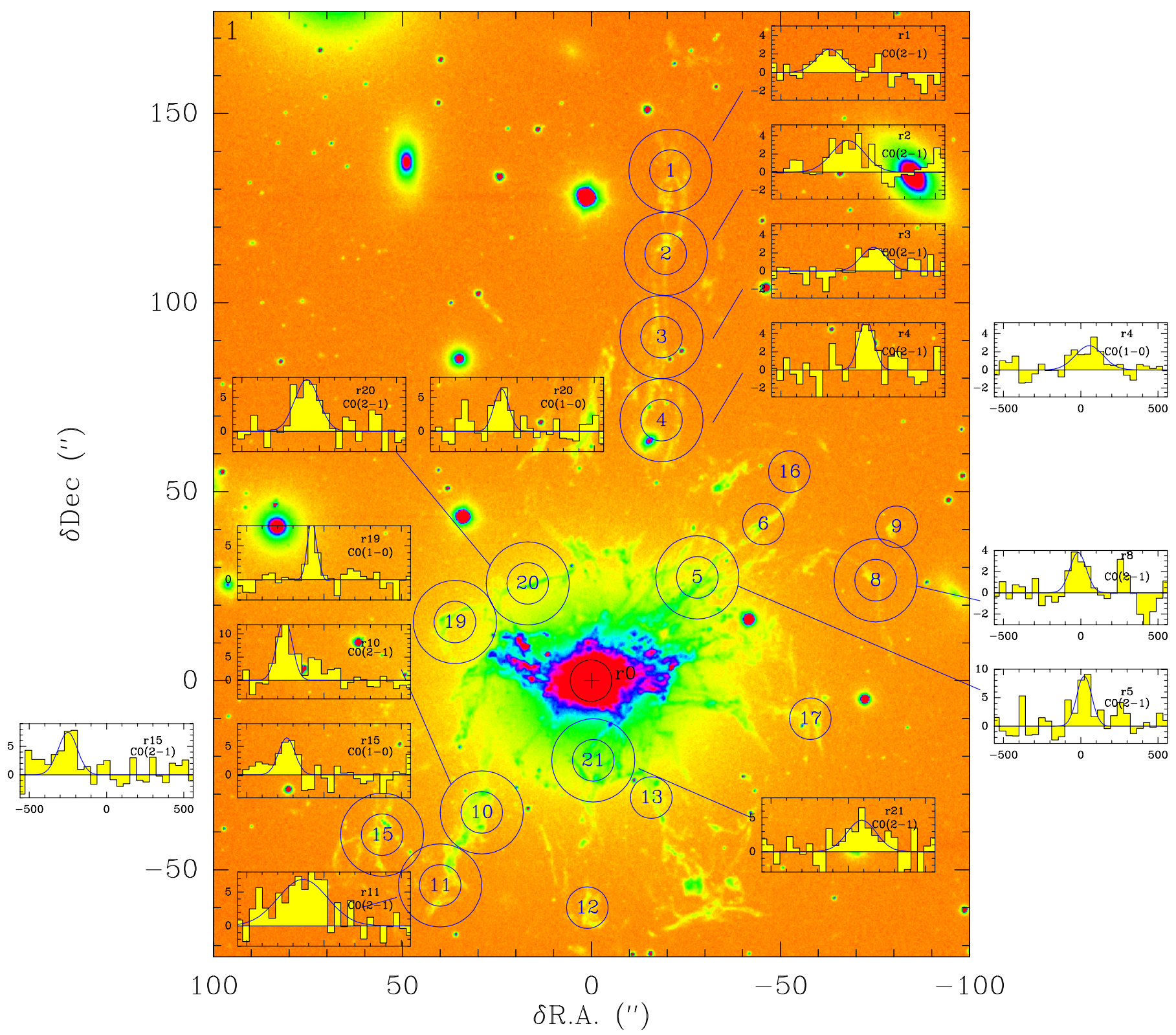

Fig. 1. CO spectra superposed on the H $\alpha$ filament structure around NGC 1275 (Conselice et al. 2001). The double circles show the 1.3 and 3 mm beamwidths at the regions where $\mathrm{CO}$ was detected. $\mathrm{CO}(1-0)$ and $\mathrm{CO}(2-1)$ spectra are shown when detected. The channel width is $42 \mathrm{~km} \mathrm{~s}^{-1}$ and the $y$-axis is main beam brightness temperature, in $\mathrm{mK}$. The velocity scale is from -560 to $560 \mathrm{~km} \mathrm{~s}^{-1}$ in each insert. Small circles $(1.3 \mathrm{~mm}$ beamwidth) show regions where no CO was detected. See Table 1 for more details.

$250 \times 4 \mathrm{MHz}$ filters for the $1.3 \mathrm{~mm}$ receivers, thereby covering $1300 \mathrm{~km} \mathrm{~s}^{-1}$ at both wavelengths. We used wobbler switching, and checked the pointing on $3 \mathrm{C} 84$, the radio source at the centre of NGC 1275. We observed in excellent weather with $T_{\text {sys }}$ typically $250 \mathrm{~K}$ at $1.3 \mathrm{~mm}$. To study the large extent of molecular gas around NGC 1275, we observed 18 regions inside the $\mathrm{H} \alpha$ filaments (Fig. 1). The beams of the $30 \mathrm{~m}$ telescope, at $3 \mathrm{~mm}$ and $1.3 \mathrm{~mm}$, are $22^{\prime \prime}$ and $11^{\prime \prime}$ respectively (corresponding to 7.7 and $3.8 \mathrm{kpc}$ at NGC 1275). The line intensities are given in mainbeam brightness temperatures, $T_{\mathrm{mb}}$. The ratio of $T_{\mathrm{mb}}$ to $T_{\mathrm{a}}^{*}$ at the $30 \mathrm{~m}$ is $T_{\mathrm{mb}} / T_{\mathrm{a}}^{*}=F_{\mathrm{eff}} / B_{\mathrm{eff}}=1.27$ at $3 \mathrm{~mm}$ and 1.75 at $1.3 \mathrm{~mm}$ (see http://www.iram.es/).

The data were calibrated with the MIRA software and reduced with the CLASS package. We dropped spiky channels and bad scans, and subtracted linear baselines from each spectrum before averaging. Table 1 summarises the results.

\section{Results, by region}

\subsection{The gas masses}

For comparison with other work, the gas mass was estimated from a standard Milky Way conversion factor $M_{\mathrm{gas}} / L_{\mathrm{CO}}^{\prime}=4.6 M_{\odot}\left(\mathrm{K} \mathrm{km} \mathrm{s}^{-1} \mathrm{pc}^{2}\right)^{-1}$ (Solomon et al. 1997) for both transitions. Note however that the ICM metallicity is known to be less than solar $Z<0.6 Z_{\odot}$ in the Perseus cluster core (Schmidt et al. 2002). This low metallicity would lead to a larger $N\left(\mathrm{H}_{2}\right) / \mathrm{CO}$ conversion factor than the standard one used here (Leroy et al. 2009) and thus under-estimated gas masses. The CO luminosity is defined by $L_{\mathrm{CO}}^{\prime}=T_{\mathrm{b}} \Delta V \Omega_{\mathrm{S}} D_{\mathrm{A}}^{2}$ with $T_{\mathrm{b}} \Omega_{\mathrm{S}}$ the source brightness temperature times the source solid angle, $\Delta V$ the line width and $D_{\mathrm{A}}^{2}$ the angular distance of the source. This gives $L_{\mathrm{CO}}^{\prime}=23.5 \Omega_{\mathrm{s} * \mathrm{~b}} I_{\mathrm{CO}} D_{\mathrm{A}}^{2}(1+z)$, with $z$ the source redshift, $\Omega_{\mathrm{s} * \mathrm{~b}}$ the solid angle of the source convolved with the telescope 
Table 1. CO detections and tentative detections, or $3 \sigma$ upper limits.

\begin{tabular}{|c|c|c|c|c|c|c|c|}
\hline Site & $\begin{array}{l}\text { Offsets } \\
\text { "X" }\end{array}$ & $\begin{array}{l}\mathrm{CO} \\
\text { line }\end{array}$ & $\begin{array}{l}T_{\mathrm{mb}} \\
\mathrm{mK}\end{array}$ & $\begin{array}{c}V \\
\mathrm{~km} \mathrm{~s}^{-1}\end{array}$ & $\begin{array}{c}\Delta V \\
\mathrm{~km} \mathrm{~s}^{-1}\end{array}$ & $\begin{array}{c}I_{\mathrm{CO}} \\
\mathrm{Kkm} \mathrm{s}^{-1}\end{array}$ & $\begin{array}{c}M_{\text {gas }} \\
10^{8} M_{\odot}\end{array}$ \\
\hline \multicolumn{8}{|c|}{ Northern Filament } \\
\hline r1 & $-21.0 \times 134.9$ & $\mathrm{CO}(2-1)$ & $2.5 \pm 1.2$ & $-184.7 \pm 28.2$ & $198.9 \pm 52.9$ & $0.5 \pm 0.1$ & 0.3 \\
\hline r1 & & $\mathrm{CO}(1-0)$ & $<3 \times 0.6$ & - & - & - & - \\
\hline $\mathrm{r} 2$ & $-19.7 \times 112.9$ & $\mathrm{CO}(2-1)$ & $3.5 \pm 1.2$ & $-65.3 \pm 23.4$ & $246.4 \pm 43.4$ & $0.9 \pm 0.2$ & 0.5 \\
\hline $\mathrm{r} 2$ & & $\mathrm{CO}(1-0)$ & $<3 \times 1$ & - & - & - & - \\
\hline r3 & $-18.6 \times 90.9$ & $\mathrm{CO}(2-1)$ & $2.6 \pm 1.2$ & $100.2 \pm 24$ & $187.6 \pm 48$ & $0.5 \pm 0.1$ & 0.3 \\
\hline r3 & & $\mathrm{CO}(1-0)$ & $<3 \times 1$ & - & - & - & - \\
\hline $\mathrm{r} 4$ & $-18.6 \times 68.9$ & $\mathrm{CO}(2-1)$ & $5.7 \pm 1.5$ & $51.3 \pm 12.6$ & $112.9 \pm 23.3$ & $0.7 \pm 0.1$ & 0.4 \\
\hline $\mathrm{r} 4$ & & $\mathrm{CO}(1-0)$ & $2.7 \pm 0.8$ & $55.2 \pm 19.4$ & $209.3 \pm 38.8$ & $0.6 \pm 0.1$ & 1.4 \\
\hline \multicolumn{8}{|c|}{ South East Filament } \\
\hline $\mathrm{r} 10$ & $28.8 \times-34.9$ & $\mathrm{CO}(2-1)$ & $13.6 \pm 2$ & $-255.8 \pm 8$ & $114.5 \pm 23.2$ & $1.7 \pm 0.2$ & 1 \\
\hline r10 & & $\mathrm{CO}(1-0)$ & $<3 \times 2$ & - & - & - & - \\
\hline $\mathrm{r} 11$ & $39.8 \times-54.2$ & $\mathrm{CO}(2-1)$ & $6.9 \pm 1.7$ & $-137.5 \pm 19.4$ & $373.1 \pm 47.6$ & $2.7 \pm 0.3$ & 1.6 \\
\hline $\mathrm{r} 11$ & & $\mathrm{CO}(1-0)$ & $<3 \times 1.8$ & - & - & - & - \\
\hline $\mathrm{r} 15$ & $55.2 \times-40.8$ & $\mathrm{CO}(2-1)$ & $7.5 \pm 2.4$ & $-250.6 \pm 21.6$ & $146.8 \pm 67.2$ & $1.2 \pm 0.4$ & 0.7 \\
\hline $\mathrm{r} 15$ & & $\mathrm{CO}(1-0)$ & $6.6 \pm 1.7$ & $-241.4 \pm 15.5$ & $123.5 \pm 30.7$ & $0.9 \pm 0.2$ & 2 \\
\hline
\end{tabular}

\begin{tabular}{|c|c|c|c|c|c|c|c|}
\hline \multicolumn{8}{|c|}{ North East Tangential Filament } \\
\hline r19 & $35.9 \times 15.5$ & $\mathrm{CO}(2-1)$ & $<3 \times 1.7$ & - & - & - & - \\
\hline r19 & & $\mathrm{CO}(1-0)$ & $8.9 \pm 1.3$ & $-77.7 \pm 5.4$ & $68.8 \pm 10.7$ & $0.7 \pm 0.1$ & 1.5 \\
\hline $\mathrm{r} 20$ & $16.7 \times 25.7$ & $\mathrm{CO}(2-1)$ & $7.7 \pm 1.7$ & $-79.8 \pm 14.6$ & $184.7 \pm 28.6$ & $1.5 \pm 0.2$ & 0.9 \\
\hline r20 & & $\mathrm{CO}(1-0)$ & $6.4 \pm 1.6$ & $-100.4 \pm 12.2$ & $109.4 \pm 24.3$ & $0.7 \pm 0.2$ & 1.7 \\
\hline \multicolumn{8}{|c|}{ Horseshoe Filament } \\
\hline r5 & $-28.1 \times 27.3$ & $\mathrm{CO}(2-1)$ & $9.1 \pm 2.3$ & $23.5 \pm 13.6$ & $101.5 \pm 58.0$ & $1.0 \pm 0.3$ & 0.6 \\
\hline r5 & & $\mathrm{CO}(1-0)$ & $<3 \times 1.6$ & - & - & - & - \\
\hline r6 & $-45.6 \times 41.4$ & $\mathrm{CO}(2-1)$ & $<3 \times 2.7$ & - & - & - & - \\
\hline r6 & & $\mathrm{CO}(1-0)$ & $<3 \times 2$ & - & - & - & - \\
\hline r8 & -75.326 .5 & $\mathrm{CO}(2-1)$ & $3.9 \pm 1.5$ & $-17.1 \pm 18.9$ & $122.1 \pm 35.2$ & $0.5 \pm 0.1$ & 0.3 \\
\hline r8 & & $\mathrm{CO}(1-0)$ & $<3 \times 0.9$ & - & - & - & - \\
\hline r9 & $-80.8 \times 40.7$ & $\mathrm{CO}(2-1)$ & $<3 \times 1.6$ & - & - & - & - \\
\hline r9 & & $\mathrm{CO}(1-0)$ & $<3 \times 0.9$ & - & - & - & - \\
\hline r16 & $-52.5 \times 55.2$ & $\mathrm{CO}(2-1)$ & $<3 \times 1.5$ & - & - & - & - \\
\hline r16 & & $\mathrm{CO}(1-0)$ & $<3 \times 1.2$ & - & - & - & - \\
\hline \multicolumn{8}{|c|}{ Southern Filament } \\
\hline $\mathrm{r} 12$ & $0.8 \times-60.1$ & $\mathrm{CO}(2-1)$ & $<3 \times 1.7$ & - & - & - & - \\
\hline $\mathrm{r} 12$ & & $\mathrm{CO}(1-0)$ & $<3 \times 1.6$ & - & - & - & - \\
\hline r13 & $-15.9 \times-30.9$ & $\mathrm{CO}(2-1)$ & $<3 \times 1.8$ & - & - & - & - \\
\hline $\mathrm{r} 13$ & & $\mathrm{CO}(1-0)$ & $<3 \times 1.3$ & - & - & - & - \\
\hline $\mathrm{r} 21$ & $-0.6 \times-21.1$ & $\mathrm{CO}(2-1)$ & $4.7 \pm 2.3$ & $86.7 \pm 37.2$ & $222 \pm 78.3$ & $1.1 \pm 0.4$ & 0.6 \\
\hline $\mathrm{r} 21$ & & $\mathrm{CO}(1-0)$ & $<3 \times 3.2$ & - & - & - & - \\
\hline \multicolumn{8}{|c|}{ Western Filament } \\
\hline r17 & $-58.0 \times-10.1$ & $\mathrm{CO}(2-1)$ & $<3 \times 1.8$ & - & - & - & - \\
\hline $\mathrm{r} 17$ & & $\mathrm{CO}(1-0)$ & $<3 \times 1$ & - & - & - & - \\
\hline \multicolumn{8}{|c|}{ Center } \\
\hline r0 & $0 \times 0$ & $\mathrm{CO}(2-1)$ & $72 \pm 6.9$ & $4 \pm 4$ & $293 \pm 10$ & $22.5 \pm 0.6$ & 15.3 \\
\hline
\end{tabular}

Notes. See Salomé et al. (2008a) for complementary data. $T_{\mathrm{mb}}=$ main-beam temperature at line peak; $V=$ velocity relative to systemic; $\Delta V=$ line width $(F W H M) ; I_{\mathrm{CO}}=$ integrated line intensity; $M_{\mathrm{gas}}(\mathrm{H}+\mathrm{He})$ is for $M / L_{\mathrm{CO}}^{\prime}=4.6 M_{\odot}\left(\mathrm{K} \mathrm{km} \mathrm{s}^{-1} \mathrm{pc}^{2}\right)^{-1}$ (Solomon et al. 1997). Note that the integration time is not the same for all regions, so the rms (computed for a spectral resolution of $42 \mathrm{~km} \mathrm{~s}^{-1}$ ) varies from one pointing to another. 

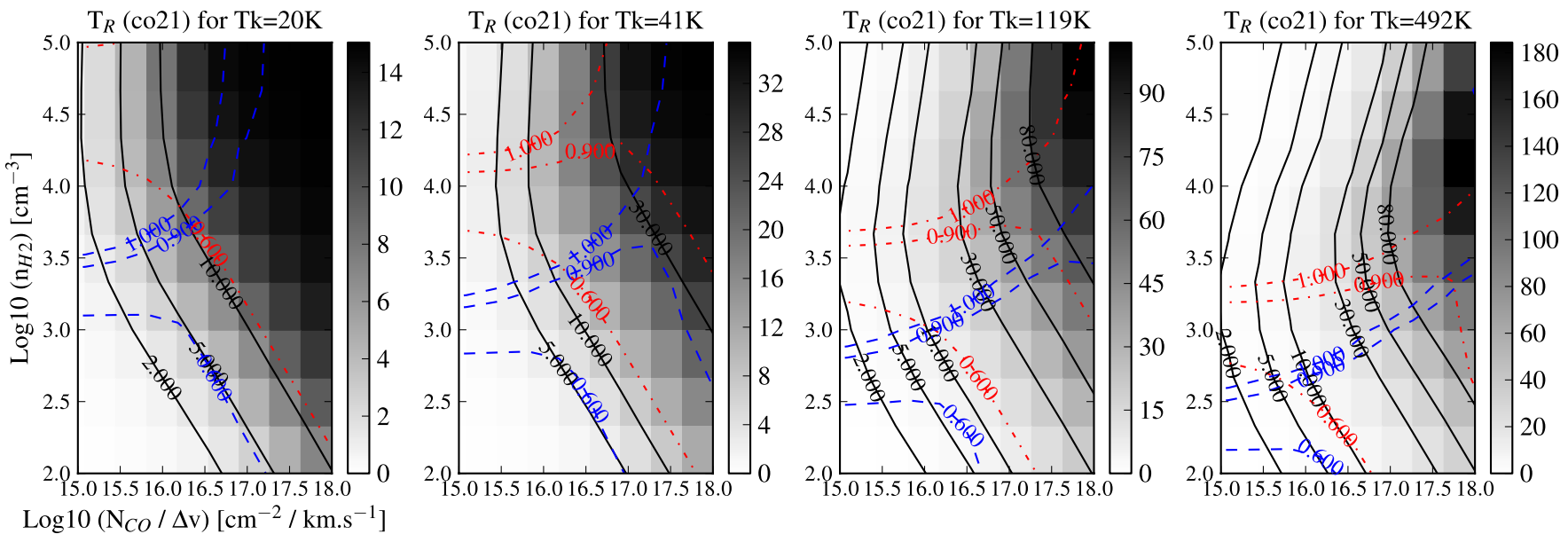

Fig. 2. Results of escape probability models from the RADEX program (Van der Tak et al. 2006) for four different kinetic temperatures, a range of $\mathrm{H}_{2}$ densities and a range of $\mathrm{CO}$ molecular column density per $\mathrm{km} \mathrm{s}^{-1}$. The grey scale mapping and the black contour lines show the expected $\mathrm{CO}(2-1)$ emission $T_{R}\left(\mathrm{co}_{21}\right)[\mathrm{K}]$ (see wedge to the right of each panel). Dashed blue lines show the $\mathrm{CO}(2-1) / \mathrm{CO}(1-0)$ line temperature ratios 0.6 , 0.9 and 1 as predicted by the RADEX program. Dash-dot red lines show the predicted $\mathrm{CO}(3-2) / \mathrm{CO}(2-1)$ line temperature ratios $0.6,0.9$ and 1. The measured $\mathrm{CO}(2-1) / \mathrm{CO}(1-0)$ line temperature ratio in $\mathrm{r} 15$ and $\mathrm{r} 20$ are close to one. So the possible solutions lie along the dashed-blue line of value one. We see that it is thus difficult to constrain the range of possible kinetic temperatures and $\mathrm{H}_{2}$ densities. Nervertheless, even with a high $T_{\text {kin }}$ of $500 \mathrm{~K}$, the density must be $\geq 10^{2.5}$. Note also that if we consider a standard Milky Way $M_{\text {gas }} / L_{\text {co }}^{\prime}$ conversion factor, then we expect $N_{\mathrm{CO}} / \Delta V>1 \times 10^{17} \mathrm{~cm}^{-2} \mathrm{~km} \mathrm{~s}^{-1}$ if $f_{2 \mathrm{D}} \sim 10^{-3}$ i.e. $T_{\mathrm{R}} \sim 10 \mathrm{~K}$, which gives slightly higher densities $\left(\geq 10^{2.9}\right)$. Finally, Bridges \& Irwin (1998) reported a $\mathrm{CO}(3-2) / \mathrm{CO}(2-1)$ line ratio close to one in the central $\sim 8 \mathrm{kpc}$ region. If this is also true for the filaments, then the possible solutions would lie at the intersection of the dashed blue line (values $\sim 0.9-1$ ) with the dash-dot red line (values $\sim 0.9-1$ ), which means even higher densities. The high- $J$ CO lines are thus important diagnostics to determine the gas properties.

beam and $I_{\mathrm{CO}}=\int T_{\mathrm{mb}} \Delta V$ the $\mathrm{CO}$ intensity ( $T_{\mathrm{mb}}$ being the main beam temperature). This is for $L_{\mathrm{CO}}^{\prime}$ in $\mathrm{K} \mathrm{km} \mathrm{s}^{-1} \mathrm{pc}^{2}, \Omega_{\mathrm{s} * \mathrm{~b}}$ in $\operatorname{arcsec}^{2}, D_{\mathrm{A}}$ in $\mathrm{Mpc}$, and $I_{\mathrm{CO}}$ in $\mathrm{K} \mathrm{km} \mathrm{s}^{-1}$. With this conversion factor, we find molecular gas masses of $\sim 3-16 \times 10^{7} M_{\odot}$ in each pointed region of the filaments. We used the same conversion factor for both transitions since line ratios were close to one. We find more mass in the (larger) $3 \mathrm{~mm}$ beam than in the $1 \mathrm{~mm}$ beam. So the $\mathrm{CO}$ emission may be extended. Note however that this is just a hint because of the large uncertainties on the line width and main beam temperature measurements for both transitions.

\subsection{The 30-kpc-long north filament, at very large radius}

This filament extends over a radial distance from 20 to $50 \mathrm{kpc}$ from the galaxy's centre. We detected $\mathrm{CO}(2-1)$ at all positions observed, but no $\mathrm{CO}(1-0)$ apart from $\mathrm{r} 4$ (Table 1, regions $\mathrm{r} 1$ to $\mathrm{r} 4)$. The fact that $\mathrm{CO}(2-1)$ is easier to detect means that the $\mathrm{CO}(1-0)$ is strongly affected by beam dilution, and therefore the emission is fragmented and clumpy on a scale of a few $\mathrm{kpc}$ (in region $\mathrm{r} 4$, where both lines are detected, the $\mathrm{CO}(2-$ 1)/ $\mathrm{CO}(1-1)$ main beam temperature ratio is $\sim 2)$. The $\mathrm{CO}(2-1)$ line centre changes from $-184 \mathrm{~km} \mathrm{~s}^{-1}$ (at $\mathrm{r} 1$ ), to $-65 \mathrm{~km} \mathrm{~s}^{-1}$ (at $\mathrm{r} 2$ ), then jumps to $+100 \mathrm{~km} \mathrm{~s}^{-1}(\mathrm{r} 3)$, and $+50 \mathrm{~km} \mathrm{~s}^{-1}(\mathrm{r} 4)$. These line velocities show the same trend as in $\mathrm{H} \alpha$ (Hatch et al. 2006). Figure 4 (middle panel) shows the observed position-velocity diagram along this filament. There is a positive gradient from $-200 \mathrm{~km} \mathrm{~s}^{-1}$ to $+100 \mathrm{~km} \mathrm{~s}^{-1}$ as the radius decreases from 50 to $35 \mathrm{kpc}$, after which the velocity falls back to $40 \mathrm{~km} \mathrm{~s}^{-1}$ at a radius of $25 \mathrm{kpc}$. The good agreement with the $\mathrm{H} \alpha$ suggests that the $\mathrm{CO}$ traces most of the mass in the filaments, possibly surrounded by ionised gas radiating in $\mathrm{H} \alpha$, at the interface with the hot intracluster medium. The shape of the gradient and the velocity reversal at the end could be due to the projection of the curved filament. Judging from the other loops that are oriented more face-on, it is obvious that many of the filaments have a significant curvature. The line width of regions $\mathrm{r} 1$ to $\mathrm{r} 3$ is $200 \mathrm{~km} \mathrm{~s}^{-1}$ while region $\mathrm{r} 4$ shows a narrower line width of $100 \mathrm{~km} \mathrm{~s}^{-1}$, more typical of the substructures in the eastern filament (see Salomé et al. 2008b). With the standard Milky Way conversion factor described above, we find a molecular gas mass of $\sim 3-14 \times 10^{7} M_{\odot}$ in the northern filament.

\subsection{The bright southeast filaments}

Regions r10, r11 and r15 (the "blue-loop") cover the southeast $\mathrm{H} \alpha$ filaments. Regions $\mathrm{r} 10$ and $\mathrm{r} 11$ are only detected in $\mathrm{CO}(2-1)$, while region $\mathrm{r} 15$ is also detected in $\mathrm{CO}(1-0)$. This filament has only negative velocities. There is a velocity change from $-130 \mathrm{~km} \mathrm{~s}^{-1}$ to $-250 \mathrm{~km} \mathrm{~s}^{-1}$ between regions r11 and r10 (compatible with the velocities of a falling filament). Region $\mathrm{r} 11$ has a broad $\mathrm{CO}(2-1)$ line width $\left(370 \mathrm{~km} \mathrm{~s}^{-1}\right)$, while in region $\mathrm{r} 10$, the $\mathrm{CO}(2-1)$ profile is narrow $\left(120 \mathrm{~km} \mathrm{~s}^{-1}\right)$. Slightly farther east is the region $\mathrm{r} 15$ at a velocity of $-130 \mathrm{~km} \mathrm{~s}^{-1}$. These southeast $\mathrm{H} \alpha$ filaments are wider than the Northern ones, and the $\mathrm{CO}$ signal is probably less affected by beam dilution.

\subsection{The north-east tangential filament}

This filament is not radially extended like most of the other filaments around NGC 1275, but is instead perpendicular to a line through the centre of the galaxy. Region $\mathrm{r} 19$ is detected in $\mathrm{CO}(1-$ $0)$ and region $\mathrm{r} 20$ in both $\mathrm{CO}(1-0)$ and $\mathrm{CO}(2-1)$. The line velocities are all negative, with no clear gradient. The line widths are narrower $\left(70,100\right.$ and $\left.180 \mathrm{~km} \mathrm{~s}^{-1}\right)$ than those of the other detections. 


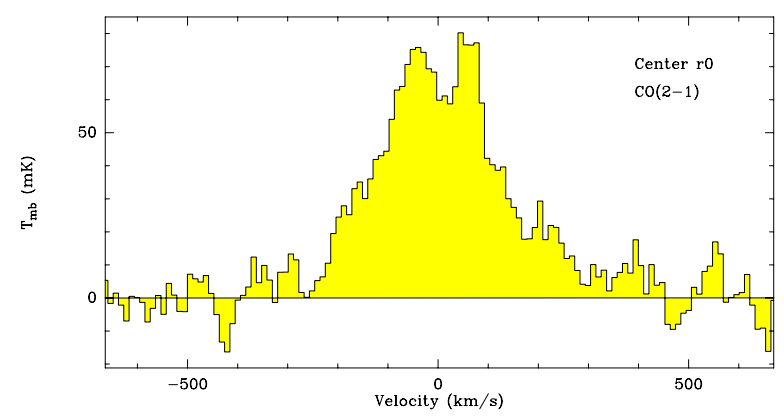

Fig. 3. $\mathrm{CO}(2-1)$ at the position of $3 \mathrm{C} 84$. The axes are main-beam brightness temperature (in $\mathrm{mK}$ ) vs. velocity, relative to $226.559 \mathrm{GHz}$ $(z=0.01756)$. The channel width is $5 \mathrm{~km} \mathrm{~s}^{-1}$.

\subsection{The horseshoe: the faintest region}

The horseshoe was re-observed in different regions (r5, r6, r8, r9, r16) all along both sides of the U-shaped loop. We clearly detect the $\mathrm{CO}(2-1)$ line in the trunk of the filament, but this is the only firm detection. $\mathrm{CO}(2-1)$ is tentatively detected in region $\mathrm{r} 8$. The other regions were not detected in $\mathrm{CO}(1-0)$ or $\mathrm{CO}(2-1)$. Surprisingly, this region has weaker $\mathrm{CO}$ emission than the other filaments. If the inclination of the horseshoe filament along the line of sight is large, then the regions we observe could be at galactocentric distances as far as the faint regions detected in the Northern filament $(50 \mathrm{kpc})$. In such a case, the small amount of molecular gas would not be visible with the rms noise we reached for those regions. The only exception is in region $\mathrm{r} 8$ where the integration time was longer (see Table 1).

\subsection{Gas in the central $4 \mathrm{kpc}$}

Figure 3 shows a double-peaked $\mathrm{CO}(2-1)$ line at the position of $3 \mathrm{C} 84$ in $5 \mathrm{~km} \mathrm{~s}^{-1}$ channels in a $4-\mathrm{kpc}$ diameter region. The negative velocities peak at $-45 \mathrm{~km} \mathrm{~s}^{-1}$ and the positive ones at $+65 \mathrm{~km} \mathrm{~s}^{-1}$. This feature can be interpreted as (i) inflowing gas, (ii) outflowing gas or (iii) a rotating pattern as observed in lines in the NIR by Wilman et al. (2005). It is not possible to disentangle from this three possibilities with the present observations alone. However, in previous observations (Salomé et al. 2006), the absence of a larger-scale rotation pattern was pointed out (inside a 8-kpc diameter region).

The double-peaked line cannot therefore be unambiguously interpreted as evidence for a central rotating disk. Instead, the large accumulation of molecular gas (nearly $10^{9} M_{\odot}$ ) in the central 4-kpc diameter of the galaxy could be the result of the recycling of cold gas inflow from the filaments back into the central potential well over several Gyr.

\section{Discussion}

\subsection{Large velocity dispersions}

Observations by Salomé et al. (2008a) showed CO line widths of 10 to $30 \mathrm{~km} \mathrm{~s}^{-1}$ in the eastern filament when observed with a $3^{\prime \prime}$ beam. In our $22^{\prime \prime}$ beam, however, the $\mathrm{CO}(1-0)$ line width in that same filament is close to $100 \mathrm{~km} \mathrm{~s}^{-1}$. In the current data from the $30 \mathrm{~m}$ telescope, most of the $\mathrm{CO}(1-0)$ and (2-1) lines have widths of order $200 \mathrm{~km} \mathrm{~s}^{-1}$ in beams of 4 to $8 \mathrm{kpc}$. In the northern filament, the velocity dispersion is even larger. These large line widths are difficult to explain by motions within a single filament. It is more likely that in our beam, we observe an ensemble of very thin sub-filaments (as observed in the optical with the
HST by Fabian et al. 2008) that consist of clumps of molecular clouds. Furthermore, these individual sub-filaments are very likely to have different projection angles, which would also increase the observed line widths, when observed with a larger beam. Nevertheless, region $\mathrm{r} 11$ has a particularly broad line width $\left(373 \mathrm{~km} \mathrm{~s}^{-1}\right)$. More observations with a larger bandwith coverage, as provided now by EMIR (Eight Mixer receiver) ${ }^{1}$ on the IRAM 30 m-telescope, would be useful to constrain with more confidence the effect of baseline fluctuation to which the results are very sensitive in the low signal/noise regime.

\subsection{Comparison with numerical models}

In Fig. 4, we compare the properties of the $\mathrm{CO}$ in the northern filament with the prediction of models in which the molecular gas forms out of uplifted ambient gas trapped by a plasma bubble. We have modelled the AGN feedback by instantaneously generating bubbles of gas in the ICM as already done by several authors (see Brüggen \& Kaiser 2001, for instance). We have used very-high resolution TreeSPH simulations combined with a multiphase model and a model of plasma bubbles. We have also taken into account the presence of cooler and denser around the radio bubbles, assuming that it is the result of ambient gas pushed out by the inflating bubble. In our simulations, a fraction of the 1-2 keV gas present at the centre of clusters is trapped and entrained by the rising buoyant bubble to higher radius where the AGN heating is less efficient. The radiative cooling then makes it cool, thereby forming cold filamentary structures in the wake and in the rim of the bubbles (see Revaz et al. 2008, for a detailed description of the model). We tried to fit the data presented here by the model number 2 of Revaz et al. (2008). This is at $t=260 \mathrm{Myr}$ and for a filament that is inclined by $60^{\circ}$ with respect to the line of sight (the top of the filament pointing towards the observer). The model reproduces not only the filament elongation, but also the mass of molecular gas detected. At radii $\leq 40 \mathrm{kpc}$, the mean velocities and the velocity dispersions are also in good agreement, but at larger radius, the model fails to reproduce the negative velocities and most of the time it underestimates the velocity dispersions by a factor of $1.5,2$ and 1.3 for regions $\mathrm{r} 1, \mathrm{r} 2$, and $\mathrm{r} 3$ respectively. The negative velocities can be understood if the gas is still being uplifted by the rising bubble. In the simulation however, at temperature below $10^{6} \mathrm{~K}$, the cooling gas decouples from the ambient gas and falls towards the centre. This discrepancy would be removed if another physical process, like viscosity or magnetic fields (Fabian et al. 2008) increase the coupling between the different gas phases.

\subsection{Line ratios}

Among the 12 regions detected here, only three have been detected in $\mathrm{CO}(1-0)$ and $\mathrm{CO}(2-1)$. All the others are detected in $\mathrm{CO}(2-1)$ but not in $\mathrm{CO}(1-0)$. One region is detected in $\mathrm{CO}(1-$ $0)$ and not in $\mathrm{CO}(2-1)$. As already mentioned, this could be explained by effects of beam dilution that makes the $\mathrm{CO}(1-0)$ emission from a clumpy molecular gas more difficult to detect than the $\mathrm{CO}(2-1)$ emission in general. Nevertheless, when closer to the centre, the filaments are widespread and overlap both the $3 \mathrm{~mm}$ and $1 \mathrm{~mm}$ beams. However, the emission seen in each wavelength may not come from the same part of the filaments. Evidence for that is the different line shapes seen in region $\mathrm{r} 4$, indicating $\mathrm{CO}(1-0)$ and $\mathrm{CO}(2-1)$ emission from dynamically and

\footnotetext{
1 http://wWw. iram.es/IRAMES/mainWiki/ EmirforAstronomers
} 

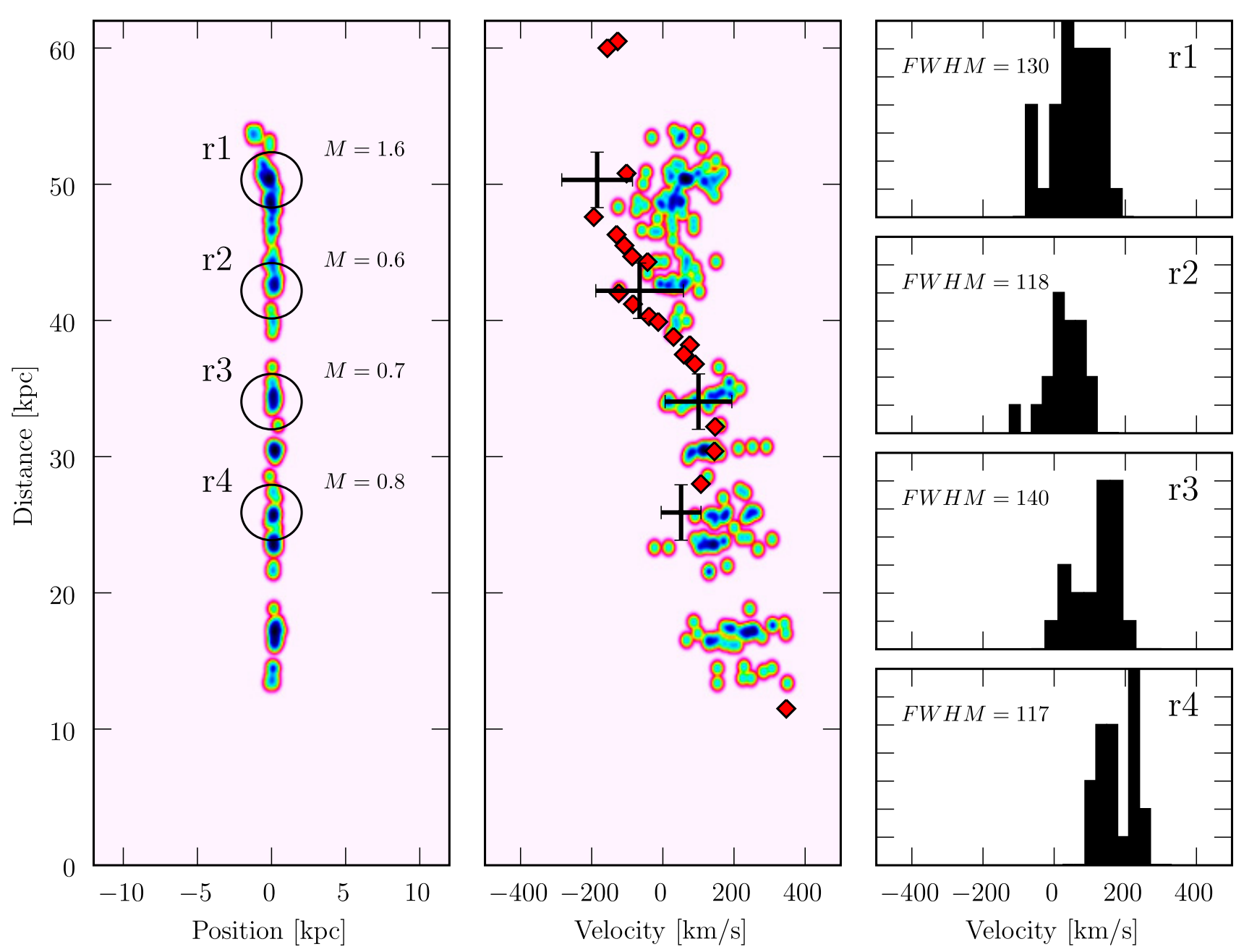

Fig. 4. Northern filament: comparison of the model with the observations. Left: contours are the surface density of molecular gas, predicted from the model. $\mathrm{CO}(2-1)$ beamwidths and positions are shown by black circles. Gas masses in each beam, predicted from the model, are in units of $10^{8} M_{\odot}$. Middle: position-velocity diagram. Pink and blue isophotes in the colour map are the model predictions. $\mathrm{Black}$ crosses are $\mathrm{CO}(2-1)$ observations. Horizontal bars are the FWHM line widths. Red diamonds are H $\alpha$ data (Hatch et al. 2006). Right: velocity distribution of the gas extracted from each region of the model. The predicted FWHM line widths of the distributions are in $\mathrm{km} \mathrm{s}^{-1}$.

spatially different regions. This also explains the clear $\mathrm{CO}(1-$ 0 ) detection alone in region r19: the region that dominates the emission lies in the $\mathrm{CO}(1-0)$ beam but not in the $\mathrm{CO}(2-1)$ one. In conclusion, the $\mathrm{CO}(2-1)$ to $(1-0)$ line ratios are very uncertain, as are the parameters derived from these values.

The three regions with best signal-to-noise ratios ( $\mathrm{r} 4, \mathrm{r} 15$, and r20) were detected in both $\mathrm{CO}(1-0)$ and (2-1). Averaged over these three regions, the $I_{\mathrm{CO}}$ and $T_{\mathrm{mb}}(2-1 / 1-0)$ ratios are both equal to $1.5 \pm 0.3$. Because interferometer maps (Salomé et al. 2008b; Lim et al. 2008) show the CO is extended in the direction of the filaments, the different beam areas at $\mathrm{CO}(1-0)$ and (2-1) mean that the apparent line ratios should be corrected downward by a factor of 2 , so that the corrected $I_{\mathrm{CO}}$ and $T_{\mathrm{mb}}$ ratios are close to 0.8 , that is, of the order of unity.

Simulations with the line escape probability program RADEX (van der Tak et al. 2007), indicate that these observed $\mathrm{CO}(2-1) / \mathrm{CO}(1-0)$ line ratios of order unity can be obtained for gas kinetic temperatures ranging from 20 to $500 \mathrm{~K}$, for $\mathrm{H}_{2}$ gas densities $>10^{2.5-3} \mathrm{~cm}^{-3}$ and $N_{\mathrm{CO}} / \Delta V>10^{15} \mathrm{~cm}^{-2} \mathrm{~km} \mathrm{~s}^{-1}$ (right panel of Fig. 2). So this gives very poor constraints on the gas properties.
With the JCMT, Bridges \& Irwin (1998) observed $\mathrm{CO}(3-2)$ emission from the central 21-arcsec region of NGC 1275 and marginally detected the ${ }^{13} \mathrm{CO}(2-1)$ inside a $22^{\prime \prime}$ beam. They made a two-component LVG model to reproduce the ${ }^{12} \mathrm{CO}(3-2) /{ }^{12} \mathrm{CO}(2-1)=1.25 \pm 0.25$ and ${ }^{12} \mathrm{CO}(2-$ 1) $/{ }^{12} \mathrm{CO}(1-0)=0.74 \pm 0.11$ line ratios. Their best model is for a cold component $(10 \mathrm{~K})$ at a density of $10^{3} \mathrm{~cm}^{-3}$ and a hot $(170 \mathrm{~K})$ component at a density of $3.1 \times 10^{4} \mathrm{~cm}^{-3}$. Furthermore, new interferometer observations at $\mathrm{CO}(3-2)$ (Salomé et al., in prep.) indicate that the $\mathrm{CO}(3-2) / \mathrm{CO}(2-1)$ ratio in some central filaments is also of order unity. This indicates that the $\mathrm{CO}$ is certainly optically thick in the central region and that the low- $J$ CO line brightness and excitation temperatures are all close to the gas kinetic temperature.

It is not possible to definitively rule out an optically thin gas in the outer filament without $\mathrm{CO}(3-2)$ observations there. However, given that the observed (beam diluted) $\mathrm{CO}(2-1)$ brightness temperatures are about $7 \mathrm{mK}$, the area filling factor $f_{2 \mathrm{D}}$ in the $\mathrm{CO}(2-1)$ beam must be $<10^{-3}$. As a check on this reasoning, for the three filament regions ( $\mathrm{r} 4, \mathrm{r} 15$ and $\mathrm{r} 20$ ), the $\mathrm{H}_{2}$ gas masses (Col. 8 of Table 1) estimated from the observed 
CO line intensities with a standard Milky Way conversion factor, are about $10^{8} M_{\odot}$, within the beam. The beam diameter at $\mathrm{CO}(2-1)$ corresponds to $4 \mathrm{kpc}$, or $1.3 \times 10^{22} \mathrm{~cm}$, so the equivalent smoothed-out $\mathrm{H}_{2}$ density within a 4-kpc diameter sphere containing $10^{8} M_{\odot}$ of $\mathrm{H}_{2}$ gas would be $0.03 \mathrm{~cm}^{-3}$, far too low a gas density to collisionally excite the $\mathrm{CO}$ (the $\mathrm{CO}(1-0)$ and $\mathrm{CO}(2-1)$ critical densities being $3 \times 10^{3} \mathrm{~cm}^{-3}$ and $10^{4} \mathrm{~cm}^{-3}$ respectively, for a temperature of $100 \mathrm{~K}$ ).

Therefore, to reach the gas density of $>10^{3} \mathrm{~cm}^{-3}$ needed to make the low- $J \mathrm{CO}$ lines optically thick, and to give line ratios close to unity, the volume filling factor $f_{3 \mathrm{D}}$ must be $<3 \times 10^{-5}$. The corresponding area filling factor within the beam must be $<10^{-3}$, and the linear filling factor $f_{1 \mathrm{D}}=f_{2 \mathrm{D}}^{1 / 2}=f_{3 \mathrm{D}}^{1 / 3}$, perpendicular to the filaments (or along the line of sight), must be $<0.03$, relative to the 4-kpc beam size.

These upper limits on the filling factors, derived from the $\mathrm{CO}$ conversion factor and the gas density required for excitation, are thus perfectly consistent with the filling factor upper limits derived above, from the observed line temperatures and those predicted by the escape probability programs, for the observed low- $J$ line ratios. The linear filling factor of $<0.03$ implies a $\mathrm{CO}$ filament width (or CO clump size) of $<120 / \sqrt{N}$ pc, $N$ being the number of clouds inside the beam, which is also consistent with our size upper limit of $<450 \mathrm{pc}$ estimated directly from our interferometric maps (Salomé et al. 2008b).

There is therefore a coherent picture in which to interpret the observed low- $J$ CO line ratios. The key is the low filling factor, which explains the observed main-beam brightness temperatures, the high gas density needed for collisional excitation in the escape probability predictions, the fact that the low-J line ratios are of order unity, the fact that the low-J lines are optically thick, and the interferometer upper limits on the CO clump sizes.

\subsection{Molecular filament formation}

The origin of the huge molecular filaments is unknown. We discuss here two possibilities. Scenario (1): the molecular gas forms in situ, far from the galaxy's centre, from uplifted warm gas that cooled down behind rising bubbles, and eventually falls back (Revaz et al. 2008). This scenario must explain how to make the $\mathrm{H}_{2}$, which is difficult to form without dust (Ferland et al. 2009). Certainly, the bubble morphology of the filaments and X-ray cavities show that this gas must be mixed from the centre of the galaxy up to $100 \mathrm{kpc}$ outward, and some dust from the galaxy must have enriched the whole region. The presence of dust could augment $\mathrm{H}_{2}$ formation rates even in the presence of $\mathrm{X}$-rays. But the sputtering due to hot ions in the X-ray emitting gas can destroys unshielded dust in $\leq 1 \mathrm{Myr}$ (Dwek \& Arendt 1992), so if there is dust in the filaments, it must have come from the galaxy's inner regions, rather from the intracluster medium. Scenario (2): the molecular gas comes from the inner part of the galaxy, entrained and dragged out by rising bubbles. The problem in this scenario is to explain how very small and dense clouds can be uplifted inside warmer gas. This is difficult to achieve, even in a highly viscous outflow and there is no such example in any observed galaxy yet. More modelling is needed to define the limits of these different scenarios. Stronger limits on the amount of dust in the filaments would help constrain the first scenario, and modelling the momentum transfer to clumps in a viscous flow (with and without magnetic viscosity) would help constrain the second scenario.

\subsection{Star formation}

There is no evidence of strong star formation in NGC 1275 filaments. Canning et al. (2010) have identified, with the Hubble Space Telescope, only two stellar regions in the south and southeast filaments (22 kpc from the nucleus). The predominantly young stellar population is concentrated in the $\mathrm{H} \alpha$ filaments. The authors derived a lower limit of $9 \times 10^{8} M_{\odot}$ stars in blue clusters and a maximum lifetime of $\sim 0.75 \times 10^{8} \mathrm{yr}$, which gives a very large $S F R$ of $\sim 20 M_{\odot} /$ yr. We have compared the amount of molecular gas with the star formation activity in these filaments The region of interest is indicated as r15 ("Blue-loop" filament) in the present paper and fills the $\mathrm{CO}(1-0)$ beam size. We have found a molecular gas mass of $\sim 2 \times 10^{8} M_{\odot}$. So if the SFR is as large as $\sim 20 M_{\odot} / y r$ then, the amount of gas is about $20 \%$ of the amount of recently formed stars. This means that most of the gas must have already been transformed into stars during the filament dynamical time (that roughly corresponds to the age of the stars). So if the star formation is still taking place in $\mathrm{r} 15$, the star formation efficiency inside this filament is very high. Indeed, even if the amount of molecular gas has been underestimated because of the sub-solar metallicity in the filaments $Z<0.6 Z_{\odot}$, (Schmidt et al. 2002), the filaments will still appear to be locally the place of an enhanced star formation activity with a star formation efficiency closer to ULIRGs than to normal spiral galaxies. The gas uplifted by the AGN bubble expansion in the ICM is pushed and compressed. In these overdense regions, the cooling is more efficient and molecular gas forms. This reservoir could then be converted very efficiently into stars in places where large scale dynamical effects can enhance the star formation (like in spiral arms of normal galaxies). The interface between the AGN cavities and the intracluster medium seems to be the place of such processes.

\section{Conclusions}

We have detected $\mathrm{CO}$ in a very extended filamentary network around NGC 1275 . Up to about $10^{9} M_{\odot}$ of molecular gas is detected inside thin and elongated filaments at distances between $7.8 \mathrm{kpc}$ and $50 \mathrm{kpc}$ from the galaxy centre. It is possible that the massive filaments seen in $\mathrm{CO}$ are an effect of the AGN feedback. The large scale motions in the Northern filament are difficult to explain with free-fall models. An accurate study of the dynamics of the filament should take into account the viscosity of the warmer gas (some part of it being uplifted). This will help lower the model predictions of the cold gas velocities. These single dish observations do not resolve the very thin filaments as observed with the HST (Fabian et al. 2008), so we certainly also mixed several filaments and averaged their individual velocities. The CO observed line widths are large. This confirms that the emission very likely comes from substructures unresolved by the $30 \mathrm{~m}$ telescope, possibly molecular cloud complexes within the filaments. Finally we reported a velocity structure in the emitting gas within $2 \mathrm{kpc}$ of the AGN that could be a rotation disk. Whether this is the fate of the molecular gas found in very distant regions $(20-50 \mathrm{kpc})$ is still an open question.

Acknowledgements. IRAM is supported by INSU/CNRS (France), MPG (Germany) and IGN (Spain). We thank the IRAM 30 m-telescope operators for their expert help with the observing.

\section{References}

Braine, J., \& Dupraz, C. 1994, A\&A, 283, 407 Bridges, T. J., \& Irwin, J. A. 1998, MNRAS, 300, 967 
Brüggen, M., \& Kaiser, C. R. 2001, MNRAS, 325, 676

Canning, R. E. A., Fabian, A. C., Johnstone, R. M., et al. 2010, MNRAS, 405, 115

Conselice, C. J., Gallagher, J. S., \& Wise, R. F. G. 2001, AJ, 122, 2281

Cowie, L. L., Hu, E. M., Jenkins, E. B., \& York, D. G. 1983, ApJ, 272, 29

Dwek, E., \& Arendt, R. G. 1992, ARA\&A, 30, 11

Fabian, A. C., Sanders, J. S., Taylor, G. B., et al. 2006, MNRAS, 366, 417

Fabian, A. C., Johnstone, R. M., Sanders, J. S., et al. 2008, Nature, 454, 968

Ferland, G., Fabian, A. C., Hatch, N. A., et al. 2009, MNRAS, 392, 1475

Gao, Yu, \& Solomon, P. M. 2004, ApJ, 606, 271

Gao, Yu, Carilli, C. L., \& Solomon, P. M., et al. 2005, MNRAS, 358, 765

Hatch, N. A., Crawford, C. S., Johnstone, R. M., \& Fabian, A. C. 2006, MNRAS, 367,433

Inoue, M. Y., Kameno, S., Kawabe, R., et al. 1996, AJ, 111

Irwin, J. A., \& Bridges, T. J. 2001, MNRAS, 328, 359

Jaffe, W. 1990, A\&A, 240, 254
Kennicutt, R. C., Jr. 1998, ARA\&A, 36, 189

Lazareff, B., Castets, A., Kim, D. W., \& Jura, M. 1989, ApJ, 335, L13

Leroy, A. K., Bolatto, A., Bot, C., et al. 2009, ApJ, 702, 352

Lim, J., Ao, Y., \& Trun, D.-V. 2008, ApJ, 672, 252

Mirabel, I. F., Sanders, D. B., \& Kazes, I. 1989, ApJ, 340, 9

Reuter, H. P., Pohl, M., Lesch, H., \& Sievers, A. W. 1993, A\&A, 277, 21

Revaz, Y., Combes, F., \& Salomé, P. 2008, A\&A, 477, 33

Salomé, P., Combes, F., Edge, A. C., et al. 2006, A\&A, 454, 437

Salomé, P., Combes, F., Revaz, Y., et al. 2008a, A\&A, 484, 317

Salomé, P., Revaz, Y., Combes, F., et al. 2008b, A\&A, 483, 793

Schmidt, R. W., Fabian, A. C., \& Sanders, J. S. 2002, MNRAS, 337, 71

Solomon, P. M., Downes, D., Radford, S., \& Barrett, J. W. 1997, ApJ, 478, 144

Van der Tak, F. F. S., Black, J. H., Schier, F. L., Jansen, D. J., \& van Dishoeck, E. F. 2007, A\&A, 468,627

Weiss, A., Downes, D., Neri, R., et al. 2007, A\&A, 467, 995

Wilman, R. J., Edge, A. C., \& Johnstone, R. M. 2005, MNRAS, 359, 755 